British Journal of Ophthalmology, 1980, 64, 597-606

\title{
Clinical trial of the use of rotating grating patterns in the treatment of amblyopia
}

\author{
C. G. KEITH, ${ }^{1}$ E. R. HOWELL, ${ }^{2}$ D. E. MITCHELL,${ }^{2}$ AND S. SMITH ${ }^{1}$ \\ From the ${ }^{1}$ Royal Children's Hospital, Melbourne, and the ${ }^{2}$ National Vision Research \\ Institute of Australia, 386 Cardigan Street, Carlton, Victoria, Australia 3053
}

SUMMARY Recent reports suggest that the vision in amblyopia can be significantly improved by brief weekly periods of occlusion of the good eye while the child performs concentrated visuomotor tasks against a background of rotating gratings. We have conducted a controlled trial to investigate the role played by the gratings in this procedure. One group $(n=33)$ of amblyopic children viewed a series of rotating gratings during treatment, while the other group $(n=27)$ performed exactly the same visuomotor tasks against a homogeneous grey background. Particular attention was placed on the choice of tests of visual acuity in order to highlight the crowding phenomenon that is a characteristic feature of the visual loss in amblyopia. The chart selected for this purpose was the Bailey-Lovie chart in which the number of letters on each line was held constant and the letter sizes and spacing were graded in equal logarithmic steps. Some improvement in vision occurred in almost all patients in either treatment group, a finding that provides support for recent claims for the effectiveness of minimal occlusion therapy. The difference between the improvement in vision of the 2 groups, averaged across all tests, was not statistically different. On only 1 of 6 acuity scores was a statistically significant difference observed in favour of the grating treatment group. However, this was observed only on patients with better than 6/60 vision where the overall improvement was itself very small. There was no detectable difference between the 2 groups either in the rate of improvement of vision or in the tendency of the vision to be maintained or even improved after cessation of treatment. Since the patients treated with gratings improved little or no more than those in the control group, we conclude that most of the visual recovery was promoted by some other aspect(s) of the procedure.

The last few years has seen a revival of interest in the use of brief or minimal periods of occlusion for the treatment of amblyopia. ${ }^{1-3}$ A particularly promising variant on this procedure which resulted in a dramatic improvement in the visual acuity of the amblyopic eye was reported by Banks $e t$ al. ${ }^{4}$ The technique required occlusion of the nonamblyopic eye once a week for only 7 minutes, during which time the child viewed a series of rotating squarewave gratings of different periods or sizes that were located immediately behind a clear plastic plate on which the child drew or played games in order to maintain fixation on the gratings. Although this procedure involved only a slight alteration to the existing practice at Addenbrooke's Hospital, Cambridge, of having the child perform concentrated

Correspondence to Dr C. G. Keith, Royal Children's Hospital, Flemington Road, Parkville, Victoria, Australia 3052. visuomotor tasks for periods of 20 minutes while the good eye was occluded, the rationale for the treatment was very different. On the basis of current knowledge of the neural mechanisms by which the visual world is encoded by the normal visual system it was thought that the grating patterns, as they rotated, would act as potent stimuli for spatial frequency and orientationally selective cells in the visual cortex. It was conjectured that the improvement that occurred in the vision of the amblyopic eye with this treatment was due to the intense and sequential stimulation by the rotating grating patterns of the visual cortical cells maintaining functional connection with the amblyopic eye..$^{5}$

In further publications the authors ${ }^{6} 7$ reported that $73 \%$ of a sample of amblyopic patients achieved an acuity in their amblyopic eye of $6 / 12$ or better both for distance and near vision after only 3 treatments of 7 minutes' duration. The number of 
treatment sessions required to achieve the maximum improvement in acuity was very low, the average being 5 , with a range of from 1 to $40 .^{7}$ This contrasted with the much longer time course of the improvement (average 13 weeks) achieved with minimal occlusion therapy. Furthermore, it was reported that the visual acuity often tended to improve even further and not regress after the course of treatment with gratings.

Independent pilot studies conducted at the Royal Children's Hospital and at the National Vision Research Institute in Melbourne confirmed that startling improvements in vision could be produced in many cases of amblyopia by use of this procedure. Although the gratings have been stressed as the most important aspect of this procedure, other factors, acting either alone or in concert, could have contributed to the observed improvement in vision. These other possible factors include the total period of occlusion each week (treatment duration plus visual function testing duration), the intense nature of the visual tasks forced upon the amblyopic eye during the periods of occlusion, and the visually guided motor tasks engaged in by the patient during the treatment session. However, since all of these factors were operating in the earlier treatment procedures that were practised at Addenbrooke's Hospital, it seemed that the grating stimuli viewed by the patients during treatment were producing the improvement in vision. Consequently, we decided to investigate directly, in the manner described below, the role played by the rotating gratings in the new treatment procedure. At the same time we made an effort to eliminate the effects of any inadvertent bias that could be introduced by the clinicians by conducting a 'blind' trial.

\section{Materials and methods}

The study was conducted in Melbourne at the Royal Children's Hospital (RCH) and the National Vision Research Institute of Australia (NVRIA). A total of 67 children with amblyopia of various causes were randomly assigned to 1 of 2 groups of approximately equal size. Both groups received weekly treatments, one with rotating gratings and the other with a uniform grey disc; all other aspects of the treatment procedure being the same for both groups. In order to control for any inadvertent bias introduced by the clinicians the trial was conducted as a 'blind' procedure: the clinician testing each patient's visual capacities was kept unaware of the nature of the treatment applied, while the clinician that supervised the treatment was unaware of the results of the visual tests. Seven children failed to complete more than 3 treatments and so have been excluded from further consideration. The age distribution of the 60 children (33 in the test group and 27 in the control group) who completed 6 treatment sessions is displayed in Fig. 1. The mean age ( 8 years) and the range ( 5 to 14 years) were both slightly greater than the group of patients treated in Cambridge. ${ }^{6}$ The distribution of distance full-line and single letter acuities prior to commencement of treatment is shown in Fig. 3. A few patients were unable to read correctly a full line of letters at any distance; in these cases an acuity score was assigned based on the part-line score (see 'Results'). In 27 of the children $(45 \%)$ the amblyopia was associated with strabismus, while in 9 cases $(15 \%)$ it was accompanied by anisometropia (defined as a difference of $1.5 \mathrm{D}$ or more in the spherical, or $1 \mathrm{D}$ in the cylindrical refraction), and in 21 cases $(35 \%)$ it was accompanied by both strabismus and anisometropia. There was little difference in the initial visual acuities of the cases with strabismus and those with strabismus and anisometropia, but in those cases of anisometropia alone the visual acuity was always $6 / 19$ or better. In 3 cases $(5 \%)$ no cause could be found for the amblyopia, and in these the visual acuity was $6 / 12$ or better. In 43 cases $(72 \%)$ there was a history of previous surgical treatment for strabismus, or occlusion therapy, or both. There was no significant difference in the distribution of visual acuities between those cases that had been treated before with occlusion and those that were untreated.

At the initial consultation a full history of the visual problem was obtained, a full ocular examination was performed, the refraction was determined

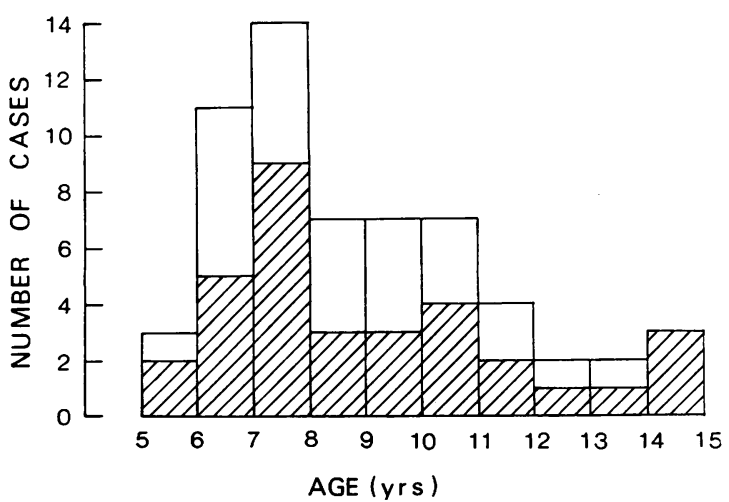

Fig. 1 Age distribution of the 60 children that completed 6 treatment sessions. Children treated with rotating gratings are depicted by hatched bars. The height of the open bars above the hatched bars depicts the number of children in each age group that viewed the grey disc during treatment. 


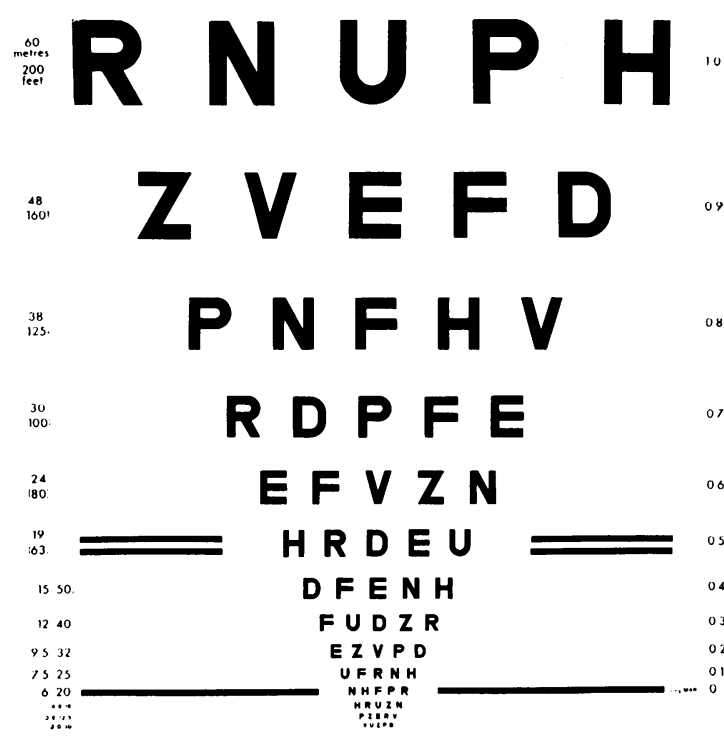

Fig. 2 An example of the Bailey-Lovie chart. ${ }^{11}$ See text for description.

under cycloplegia when indicated, and a variety of visual functions were tested as described below. Considerable thought was given to the testing of visual acuity particularly in order to score visual improvement equally for all grades of visual acuity. For this reason standard Snellen test charts were not used routinely because the differences in letter size between consecutive lines do not correspond to any regular pattern (BS 4274, British Standards Institution, 1968). Furthermore, it is likely that the contour interaction effects that are characteristic of amblyopia ${ }^{8-10}$ would not be constant for each line with such charts because of the unequal number of letters on each line and the frequent lack of a constant relative spacing of letters. In fact conventional charts contain only 1 letter of the largest size $(6 / 60)$ and 2 of the second largest (6/36), making it impossible to differentiate between the performance for single letters and that for a row of letters. For these reasons the chart (Fig. 2) described by Bailey and Lovie ${ }^{11}$ was used for determining distance vision. This is characterised by an equal number of letters ( 5 in each row) and by a regular logarithmic scaling in $0.1 \mathrm{log}$ unit of both the size and spacing of the letters of each line as well as the separation between lines. Consequently, there are 10 equally graded letter sizes from $6 / 60$ to $6 / 6$, the intervening sizes being $6 / 48,6 / 38,6 / 30,6 / 24,6 / 19,6 / 15,6 / 12$, $6 / 9 \cdot 5$, and $6 / 7 \cdot 5$. This chart highlights the contour interaction effects characteristic of amblyopia, and it has the additional advantage of simplifying the scoring of acuities of less than $6 / 60$. As the child approaches the chart by a distance corresponding to a change of $0.1 \mathrm{log}$ unit, the letter sizes alter by an equivalent amount, thus allowing easy conversion to the usual numerator of 6 . For example, on viewing the chart at 4.8 metres the $6 / 6$ letters are equivalent to $6 / 7.5$ and the top row of letters is equivalent to $6 / 75$; similarly at 3.8 metres, $6 / 95$; and at 3 metres, $6 / 120$, etc.

Characteristically amblyopes are unable to read a full line of letters below a certain level but are able to read a few smaller letters correctly, usually those at either end of a row or occasionally in the middle of a row. We therefore recorded the lowest line in which only 1 or more letters could be seen ('part' score), as well as the lowest full line of letters read ('full' score).

The acuity for single letters was measured using the Sheridan-Gardiner series of plates. These have 3 letters corresponding to each size, and are obtainable only in the conventional Snellen sizes. Separate acuities were recorded depending on whether all ('full' score) or only some ('part' score) of the letters of any one size could be read. Near visual acuity was measured at a distance of $33 \mathrm{~cm}$ by the Sheridan-Gardiner near test, which has 6 letters of each size corresponding to the usual Snellen test types. As with the other tests, full and part acuities were recorded.

Tests of stereopsis were made either by the Titmus plates alone $(\mathrm{RCH})$ or by the Titmus, Toegepast Natuurwetenschappelijk Onderzoek (TNO), and Frisby tests (NVRIA). In addition measurements of contrast sensitivities and acuity for sine-wave gratings were made on patients examined at the National Vision Research Institute in a manner described in detail elsewhere. ${ }^{12}$ The results of these particular measurements will be described in detail in a later publication (Howell, Mitchell, and Keith, in preparation).

For assessing visual acuities and for treatment the nonamblyopic eye was occluded with an opaque disc, patch, or plaster, except when there was latent nystagmus, when semitransparent tape was placed over the spectacle lens to blur vision to less than $6 / 60$.

The treatment was commenced when all the necessary data had been obtained and after any significant refractive error had been corrected (1 dioptre or more). The child was seated in front of a specially built device on which the appropriate disc could be placed immediately behind a clear plastic plate. The grating discs, which were rotated at 1 revolution per minute, were $20 \mathrm{~cm}$ in diameter and hence slightly larger than those employed in previous investigations. ${ }^{45}$ There were 6 gratings in all, the period of each differing from the next by a 
factor of 2, with the largest having a period of $2 \mathrm{~cm}$. The rotating grey disc, which was employed for the control group, was of the same diameter and had approximately the same mean luminance as the gratings.

Each grating was presented for either 1 minute $(\mathrm{RCH})$ or $1 \frac{1}{2}-2$ minutes (NVRI), so that the total treatment procedure occupied no more than 12 minutes. During this time the child played games of a competitive nature with the therapist such as noughts and crosses. Younger children, or those with very poor vision, drew figures or filled in circles of different sizes. It was intended that the child view the patterns from about $50 \mathrm{~cm}$, but many children worked at a closer distance, particularly while drawing. Those children allotted to the grey disc treatment group played exactly the same games for the same period of time.

Treatment sessions were held weekly for most

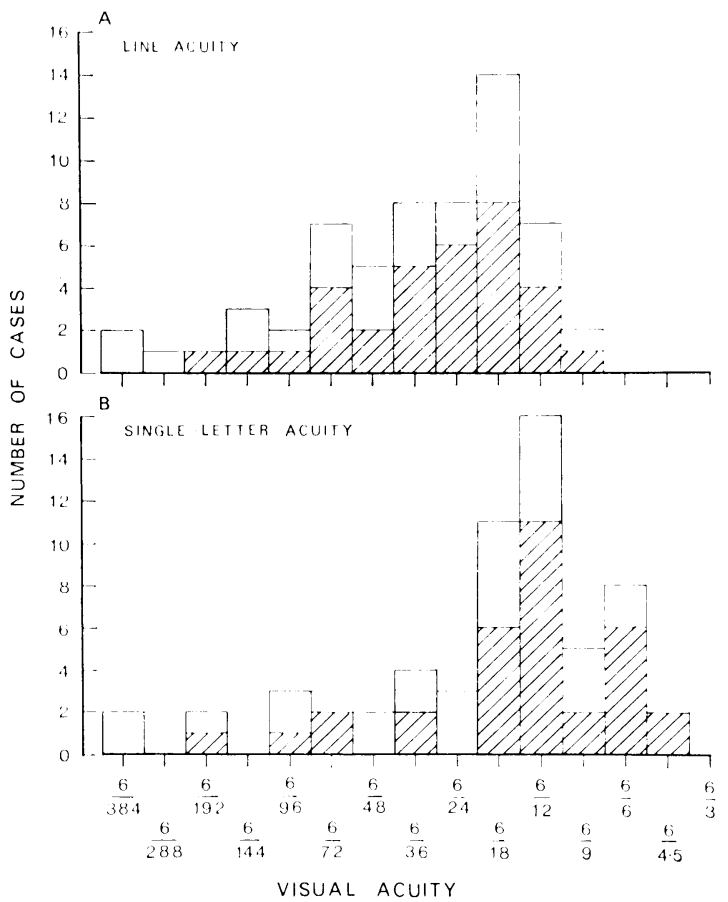

Fig. 3 Distribution of distance full-line acuities (A, top) measured on the Bailey-Lovie chart and single-letter (B, bottom) acuities of the 60 patients prior to commencement of the 6 treatment sessions. Hatched bars depict patients that viewed rotating gratings during treatment. The height of the open bars above the hatched bars indicates the number of patients from the control grey group having the various acuities indicated. 'Part' scores have been plotted for those patients who were unable to identify all the letters in any row, or all the single letters of a given size. cases, though occasionally 2 weeks elapsed between some visits. The previously described visual functions, with the exception of the measurements of grating acuity or contrast thresholds, were tested before (NVRIA) and after ( $\mathrm{RCH})$ each treatment. These tests necessitated occlusion of the nonamblyopic eye for approximately 15 minutes. The visual tests, as well as the treatment, were conducted in well illuminated surroundings, the luminance of the test charts and of the stimuli viewed during treatment was about $100 \mathrm{~cd} / \mathrm{m}^{2}$. At the conclusion of 6-weekly treatment sessions the patient was seen 1 week later for a final assessment, which included recording of all the visual functions examined prior to the initial treatment. Only on completion of the assessment was the clinician made aware of the nature of the treatment the patient received during the preceding 6 weeks. At this stage those patients who were treated with the rotating grating patterns were asked to return in 1 or 2 months for review. Seven subjects from the control (grey disc) group (NVRIA) were given an additional 6 treatment sessions with the rotating gratings.

\section{Results}

VISUAL ACUITY

Virtually all the patients from both groups, treated with either the rotating gratings or the grey disc, showed some improvement on 1 or more of the tests of visual function. The initial and final full-line acuities after 6 treatment sessions are shown in Fig. 4, while Fig. 5 displays the distance singleletter acuities before and after treatment. Fig. 6 shows the results obtained on the near visual acuity tests. In the 5 cases where no initial distance full-line acuity could be obtained the acuity was arbitrarily scored as 1 line $(0 \cdot 1 \log$ unit) worse than the partline score. The same procedure was followed in those cases where only some of the 3 single-letter plates of any given size could be identified at the outset of the trial. These particular patients are identified in Figs. 4 and 5 by boxed symbols.

It can be seen that there was no obvious difference between those children treated with the gratings and those with the grey disc, nor was there any obvious difference in the improvement in near vision in comparison with the distance vision. However, the distance vision for single letters improved slightly more than the full-line acuity. One notable feature was the dramatic improvement on all visual tests observed in a few of the patients with initial visual acuities worse than $6 / 60$ (6/60 minus group). A comparable improvement was not seen in any child whose initial vision was better than $6 / 60$ (6/60 plus group). 


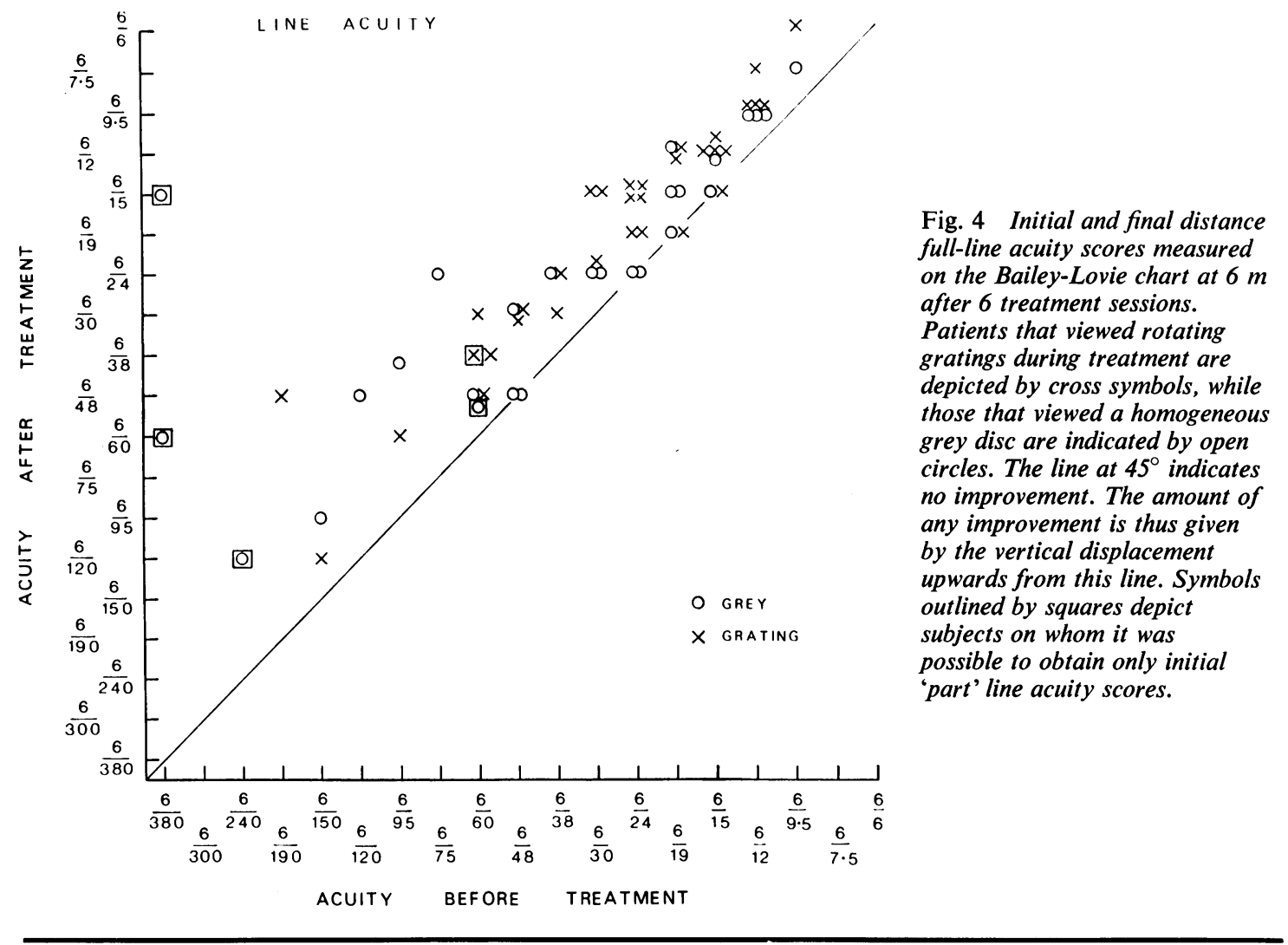

Fig. 5 The distance single letter scores before and after 6 treatment sessions. Symbols are as in Fig. 4. The amount of any improvement in vision is given by the vertical displacement upwards from the line at $45^{\circ}$. Symbols outlined by squares depict subjects on whom it was possible to obtain only 'part' scores.

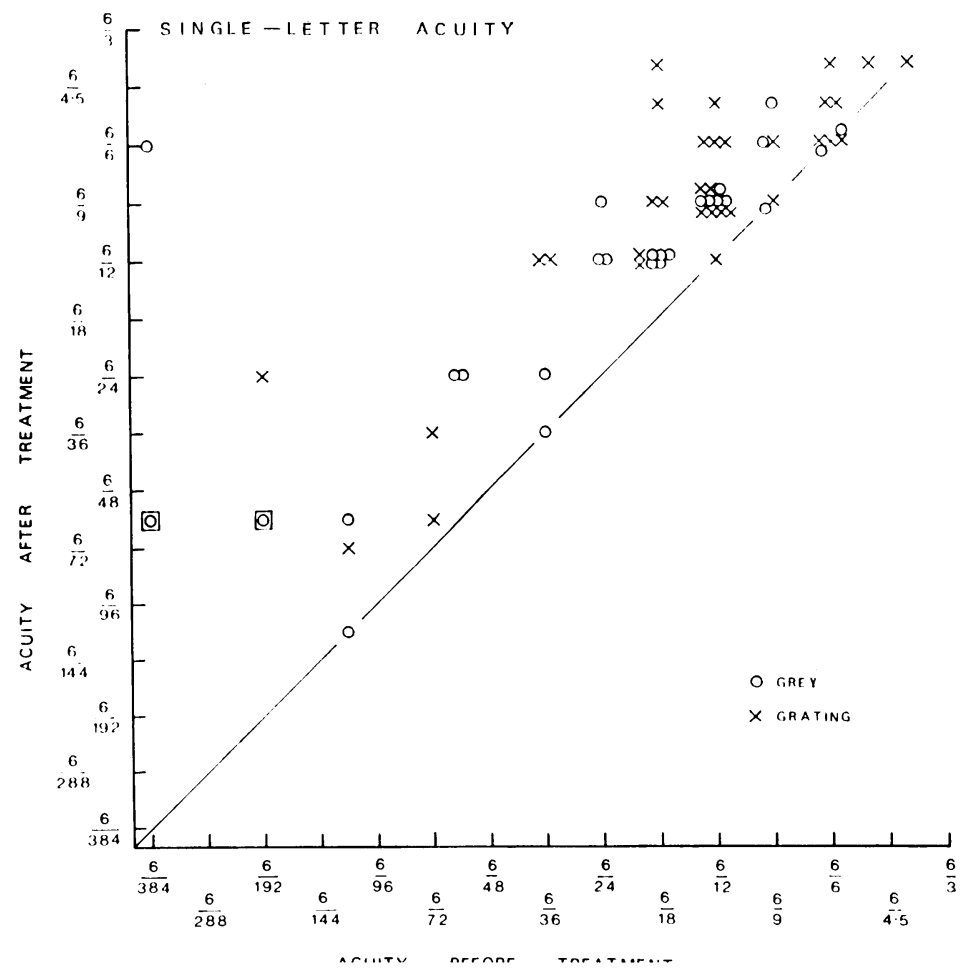


Fig. 6 Near acuity measured with the Sheridan-Gardiner near test before and after the 6 treatment sessions. Symbols are as in Fig. 4. The amount of any improvement in vision is given by the vertical displacement upwards from the diagonal line.

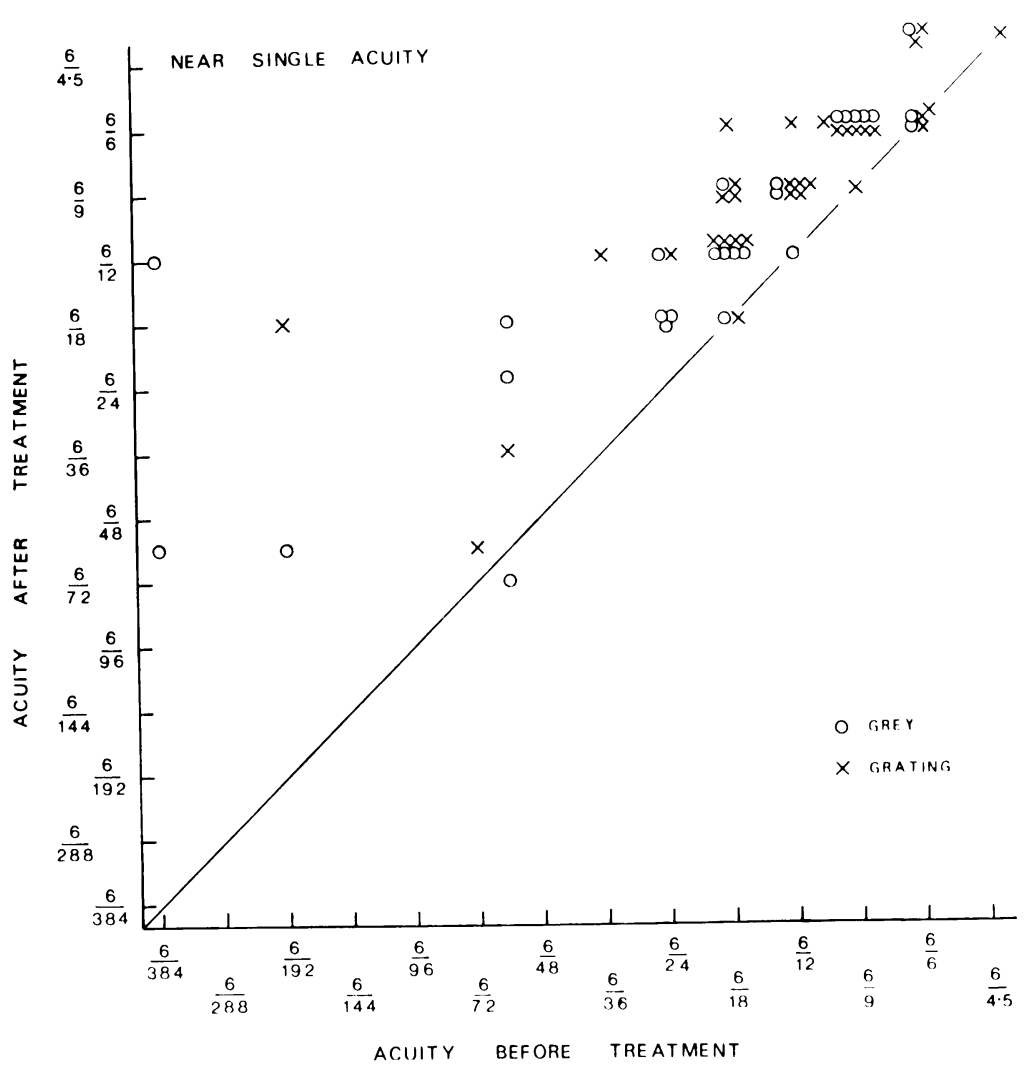

A quantitative comparison was made between the test group and control group and the results are shown in Table 1. For each subject the improvement in visual acuity between the initial and final assessment was expressed in terms of the ratio of the letter sizes on a logarithmic scale. This was made easy in the case of the distance line acuities since the rows of letters on the Bailey-Lovie chart are already scaled in equal logarithmic steps. An improvement of 1 line on this chart is equivalent to $0.1 \log$ unit, while a 3-line improvement is equivalent to $0.3 \log$ unit, or a halving of the letter size. To score the improvement in the single letter and near visual acuities, the Snellen notations were first converted to their logarithmic equivalents. Only patients on whom actual full or part acuity scores could be obtained were included in the quantitative comparison shown in Table 1. The patients were at first considered in 4 groups according to whether their initial distance full line acuities fell between the following bounds: less than $6 / 60$, between $6 / 60$ and $6 / 38$, between $6 / 30$ and $6 / 15$, or $6 / 12$ or better. However, since the degree of improvement observed in the latter three groups proved to be so similar, these results were combined for the analysis which is shown in Table 1 . In this table the mean improvement is given for each test of visual acuity: distance 'full' and 'part' line acuity, distance 'full' and 'part' single-letter acuity, and near single-letter 'full' and 'part' acuity. The standard deviation of the scores is shown adjacent in brackets for the subjects in the $6 / 60$ plus group, while only the range has been shown for the $6 / 60$ minus group since the numbers were so small.

As was apparent from Figs. 4, 5, and 6, the mean improvement in the $6 / 60$ minus group was much more marked than among the subjects with better than $6 / 60$ vision. The average improvement for the former group was $0.48 \mathrm{log}$ unit (equivalent to 4.8 lines on the Bailey-Lovie chart) for patients treated with either rotating gratings or the grey disc, while in the $6 / 60$ plus group the average improvement was only $0.17 \mathrm{log}$ unit $(1.7$ lines) for subjects treated with gratings and $0.14 \log$ unit ( 1.4 lines) for those receiving treatment with the grey disc. Within the group with vision of $6 / 60$ or better there was no obvious difference in the degree of improvement between those patients treated with gratings or the grey disc except on the distance full-line acuity measures. On this particular test the 29 patients 
Table 1 Mean improvement in acuity scores for each visual acuity test (log units)

\begin{tabular}{|c|c|c|c|c|c|c|c|}
\hline \multirow{2}{*}{ Treatment group } & \multicolumn{7}{|l|}{ Acuity test } \\
\hline & $D L F$ & $D L P$ & DSF & DSP & NSF & NSP & All tests \\
\hline \multicolumn{8}{|c|}{$6 / 60$ Minus group (range of scores in brackets) } \\
\hline Gratings & $0.33(0 \cdot 1-0 \cdot 7)$ & $0.3(0 \cdot 1-0.8)$ & $0.38(0 \cdot 1-0.9)$ & $0.5(0.2-1 \cdot 0)$ & $0.4(0 \cdot 1-1 \cdot 0)$ & $0.57(0 \cdot 2-1 \cdot 2)$ & 0.41 \\
\hline Number of cases (4) & 3 & 4 & 4 & 4 & 4 & 3 & \\
\hline Grey & $0.38(0 \cdot 2-0 \cdot 5)$ & $0.53(0.1-1.4)$ & $0 \cdot 48(0-1 \cdot 8)$ & $0.48(0 \cdot 3-0.8)$ & $0.49(0-1 \cdot 5)$ & $0.53(0-1 \cdot 6)$ & $0 \cdot 48$ \\
\hline Number of cases (8) & 4 & 8 & 6 & 6 & 7 & 6 & \\
\hline \multicolumn{8}{|c|}{$6 / 60$ Plus group (standard deviation of scores in brackets) } \\
\hline Gratings & $0.15(0.084) *$ & $0 \cdot 13(0 \cdot 1)$ & $0 \cdot 2(0 \cdot 19) \dagger$ & $0.2(0 \cdot 14)$ & $0 \cdot 18(0 \cdot 13)$ & $0.16(0.14)$ & $0 \cdot 17$ \\
\hline Number of cases (29) & 29 & 29 & 29 & 22 & 29 & 22 & \\
\hline Grey & $0.08(0.09)^{*}$ & $0.11(0.06)$ & $0 \cdot 16(0 \cdot 13) \dagger$ & $0 \cdot 18(0 \cdot 16)$ & $0.16(0.09)$ & $0 \cdot 14(0 \cdot 15)$ & $0 \cdot 14$ \\
\hline Number of cases (19) & 19 & 19 & 19 & 18 & 19 & 16 & \\
\hline
\end{tabular}

The acuity tests above were: distance line full (DLF), distance line part (DLP), distance single letters full (DLF), distance single letters part (DSP), near single letters full (NSF), and near single letters part (NSP).

*For $6 / 60$ plus group, DLF $t=2.86,0.01<P>0.001$. $\dagger$ For $6 / 60$ plus group, DSF $t=0.8,0.5<P>0.1$.

treated with gratings improved an average of $0 \cdot 15$ $\log$ unit, while those treated with the grey disc changed somewhat less $(0.08 \log$ unit). The small difference between the average improvement of the 2 treatment groups on this one particular test was, however, statistically significant at the 0.005 level on both a Student $t$ test and the nonparametric Mann-Whitney U test.

Further analysis of the data from the group of patients with better than $6 / 60$ vision revealed no obvious difference in the degree of improvement among children that had received previous occlusion therapy and those that had received no prior treatment. There was also no observable difference in the response to treatment between children from either group with different forms of amblyopia (strabismic, anisometropic, etc.). Finally, examination of the longitudinal acuity scores across the 6 treatment sessions revealed no tendency for patients in the 2 treatment groups to respond to treatment at different rates.

\section{CONTRAST THRESHOLD FUNCTIONS}

The contrast thresholds for sinusoidal gratings were measured in each eye as a function of spatial frequency for 25 patients both before and after 6 treatment sessions. These measurements provide information on the relative threshold elevation in the amblyopic eye and also enable the limit of grating resolution acuity to be assessed. In general, the degree of amblyopic loss measured by this technique did not correlate in any simple manner with the degree of visual loss displayed on letter charts. Before treatment 19 out of 25 patients had a grating acuity of 30 cycles per degree or higher (mathematically equivalent to Snellen $6 / 6$ or better). Only 2 patients had a grating acuity less than 20 cycles per degree (worse than 6/12). After treatment only 3 out of 25 patients showed a significant improvement in contrast thresholds or grating acuity. Of these, 2 were in the grey control group.

It was clear that contrast threshold function measurements do not clearly indicate the extent of the deficit as shown by letter charts, nor is it always a sensitive indicator of improvement in visual performance following treatment. As the issues involved here are rather complex, these findings will form the basis of a further publication (Howell, Mitchell, and Keith, in preparation).

\section{STEREOPSIS}

Stereoacuity was measured before and after the 6 treatment sessions in 52 of the patients. In agreement with a previous report ${ }^{13}$ measures of stereopsis obtained with the Frisby and Titmus test showed good correspondence, but those measured with the TNO test showed consistently poorer scores. Stereoacuity improved significantly in 8 out of 28 patients who were in the grating group and 5 out of 24 patients in the grey control group. In all but 1 case the final stereoacuity was better than 100 seconds, with 6 cases having clinically normal acuity of $40 \mathrm{~s}$ or better. There was no significant difference in improvement between the 2 groups. In all cases in which there was improvement fixation was central or parafoveal, with either no deviation or a squint angle less than $5^{\circ}$, and there had been no surgical intervention. These findings will be described in greater detail in a later publication.

FOLLOW-UP

Twenty patients from the $\mathrm{RCH}$ returned for a review examination between 1 and 9 months after cessation of treatment. The visual acuity was con- 
sidered unchanged if there was not more than $0 \cdot 1$ log unit difference from the score at the end of treatment. Among those patients treated with gratings 8 were unchanged, 2 had deteriorated almost to the level which existed prior to initiation of treatment, and only 1 had improved (by $0.2 \log$ unit). Of the 9 patients in the grey control group 7 remained unchanged and 2 had deteriorated to pretreatment levels. Fifteen of the patients attending the NVRIA were reviewed between 1 and 2 months after conclusion of treatment. Of 8 treated with gratings 7 were unchanged and 1 had deteriorated, while of the 7 in the control group 6 were unchanged and 1 had become worse. Thus there did not appear to be any obvious difference between the 2 treatment groups.

The comparable nature of the response of the patients to the 2 treatment regimens was also to be seen in the results from the 7 patients from the group treated with grey discs that were subsequently treated with rotating gratings. Six of these patients showed no further improvement in response to treatment with gratings, and only 1 patient improved by more than 1 line. This particular patient improved by the same amount $(0.2 \mathrm{log}$ unit) in response to the 2 treatment procedures.

\section{Discussion}

This study was primarily designed to assess the role of rotating gratings in the treatment of amblyopia by minimal occlusion. However, the results also provide insight into the value of minimal occlusion therapy as well as information on the relative effectiveness of different visual acuity tests for assessment of the degree of amblyopia. One control group was used, this group receiving as similar as possible management to the grating group but without exposure to the rotating gratings during treatment. As virtually all patients in both groups showed some improvement in vision with treatment, and the average improvement in the 2 groups were so similar, there are clearly other factors which are making significant contributions to the improvement. As these other factors were not controlled in this study, we can only speculate on their importance. Features of the treatment procedure that could contribute to the visual improvement include the period of weekly occlusion, which, including the time required for the tests of the visual function of the amblyopic eye, amounted to about half an hour; the intense nature of the visual tasks performed by the amblyopic eye during testing and treatment; and the need for concentrated eye-hand co-ordination during the treatment sessions. However, a number of other factors that might exert an influence include the nonspecific improvements that occur with any type of treatment-therapist and possibly patient enthusiasm as well as the effect of practice in performing the various visual acuity tests. Finally, in addition to the factors already listed it is possible that some improvement might be attributed to the generalised arousal of the child on the occasion of each visit to the hospital or institute. Further studies are obviously necessary in order that these factors may be isolated and their relative contribution determined.

The grating test group in general showed similar magnitude of improvement in visual acuity to the control group. Inspection of Table 1 indicates that the average improvement on all 6 acuity scores of the patients in the subgroup treated with gratings and having an initial acuity better than $6 / 60$ was marginally greater than that of the patients in the control group. However on only 1 measure, the distance full-line acuity scores on the Bailey-Lovie chart, was this very small difference statistically significant by both a Student $t$ test and the nonparametric Mann-Whitney U test. On this measure the grey control subgroup improved by $0.08 \mathrm{log}$ unit ( 0.8 of a line) and the grating subgroup by 0.15 log unit ( 1.5 lines) giving a relative improvement of $0.07 \log$ unit $(0.7$ line). This difference was not seen so clearly in the part-line and single-letter scores, where the grey control group scores were larger. In the $6 / 60$ minus subgroup the general magnitude of improvement was much greater $(0.48 \log$ unit average across all tests for the grey group) but again with no significant difference between the grating and the grey group.

Active stimulation of the amblyopic eye by intense visual stimuli has been advocated in the past for the treatment of amblyopia. ${ }^{14-16}$ The Cambridge group also regarded the visual stimuli received during treatment as an essential factor contributing to the improvement observed in their patients. It was thought that the series of rotating gratings acted as particularly potent stimuli for cells in the visual cortex receiving input from the amblyopic eye. However, since the improvement in the vision of the 2 groups of patients in our study was so similar, it would appear that aspects of the treatment procedure other than the rotating gratings were responsible for most of the improvement that was observed. However, the possibility still remains that stimulation of the amblyopic eye with rotating gratings produces a very slight further improvement in vision in some patients over that produced by the other aspects of the minimal occlusion therapy.

The visual improvement in our patients was not quite so marked as that reported by Campbell et al. ${ }^{6}$ who found that $73 \%$ of their patients achieved $6 / 12$ 
vision or better on conventional test charts, while only $28 \%$ of our treated patients reached this level on the Bailey-Lovie chart. However, some of the discrepancy between the 2 findings can be accounted for by the differences between the visual acuity tests that were employed in the 2 studies. As emphasised earlier, the measured acuity of the amblyopic eye differed according to the visual acuity test that was administered. In general, the measured acuity was best for single letters and lowest with the BaileyLovie chart. Conventional test charts, as already discussed, are characterised by both an unequal number of letters on each line and an unequal relative spacing between letters. Although there are a greater number of letters towards the bottom of the chart, the spacing is relatively larger, so that contour interaction effects are reduced on these lines in comparison with lines in the middle of the chart, where these effects are greatest. Consequently an amblyopic eye will tend to perform better on conventional charts than on those such as the BaileyLovie chart, where some attempt has been made to maintain contour interaction effects constant on each line.

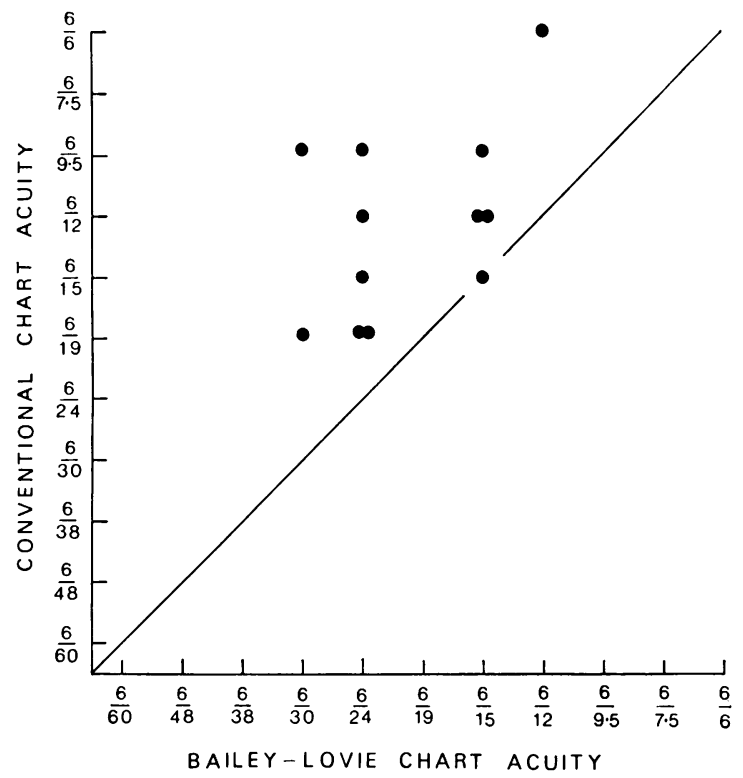

Fig. 7 The acuity scores of 12 amblyopic patients measured on both the Bailey-Lovie chart and a conventional test chart conforming to the British standard (BS 4274, British Standards Institution, 1968). To aid comparison the divisions on the 2 axes conform to the letter sizes on the Bailey-Lovie chart. The diagonal line indicates equal acuity on the 2 charts. Note that, with the exception of 1 patient, scores on the conventional chart indicated better acuity than those on the Bailey-Lovie chart.
This point is graphically illustrated in Fig. 7, which shows the relationship between the acuity scores obtained on conventional and Bailey-Lovie charts for the 12 patients on whom both these measures were made. With the exception of 1 child, on whom the scores were identical, measures obtained on the former chart were consistently better by several lines and in 1 child by 5 lines $(6 / 6$ versus 6/30). On the other hand the discrepancy between the scores of these 12 patients on the conventional acuity chart and on single-letter acuity tests were not so marked. In 5 cases the acuities were the same, and in 7 the single-letter acuity was better by about 2 lines. In agreement with this latter finding the final singleletter acuity was $6 / 12$ or better in $78 \%$ of our patients, a figure comparable to the proportion of patients $(73 \%)$ treated by Campbell et al. ${ }^{6}$ that attained this acuity on conventional test charts.

Although the patients in this study were heterogeneous for the type of amblyopia, there was no observable difference in the response to treatment between patients having different types of amblyopia. This was so despite the fact that the initial vision was quite different for the various types, being worst among strabismic amblyopes, relatively good $(6 / 19+)$ for anisometropic amblyopes, and best $(6 / 12+)$ for the small number of subjects with no observable accompanying peripheral defect.

In addition to the level of acuity eventually attained in response to treatment with rotating gratings Campbell et al. ${ }^{6}$ also placed some emphasis on the increase in the rate of improvement of vision over that achieved by minimal occlusion therapy. To produce the same degree of visual improvement (to 6/12) achieved after only 3 weekly treatment sessions with rotating gratings 13 weeks of occlusion for 20 minutes each day were required. By contrast we observed no consistent difference in the rate of visual improvement among the members of the 2 groups treated with gratings or with grey disc. Another feature of the rotating grating treatment mentioned by Banks et al. ${ }^{4}$ and later by Banks and Barnet $^{17}$ was the improvement that tended to occur after treatment was discontinued. This was not consistently observed in the present study. While 1 patient from the group treated with gratings and 3 patients from the grey control group improved in visual acuity by $0.1 \log$ unit, only 1 patient (treated with gratings) improved by more than this amount $(0.2 \log$ unit). Thus the 2 groups did not differ noticeably after cessation of treatment.

The work of the Cambridge group has stimulated renewed interest in the use of minimal occlusion in association with intensive visual tasks and concurrent visually guided motor activity in the treatment of amblyopia. We have confirmed their 
findings that reasonably substantial visual improvements can be produced by this treatment (although we cannot exclude that this is not just due to practice), which is possibly preferable to long periods of unsupervised occlusion in the older child, and in cases of latent or manifest nystagmus which are in fact aggravated by occlusion. However, we have found that overall the rotating grating patterns that were viewed during treatment made little or no contribution to the improvement in vision or to the rate at which this occurred.

We thank Mrs J. Krstic, Mrs L. Brierley, and Mr V. Stepanow, who assisted with the treatment of a proportion of the patients. We are particularly indebted to $\mathrm{Mr} \mathbf{M}$. Smith for the initial clinical assessment of the patients at the NVRIA. This study was supported by a grant (78/3807) to E.R.H. and D.E.M. from the National Health and Medical Research Council of Australia.

\section{References}

1 Stenius S. Sehübungen bei Scheilamblyopie und ihre Ergebnisse. Zentralbl Gesamte Ophthalmol 1935; 33: 683.

2 Catford GV. Amblyopic occlusion: the results of treatment. Trans Ophthaimol Soc UK 1967; 87: 179-93.

3 Cibis L. History of amblyopia and its treatment. $\mathrm{Am}$ Orthopt J 1975; 25: 54-61.

4 Banks RV, Campbell FW, Hess RF, Watson PG. A new treatment for amblyopia. Br Orthopt J 1978; 35: 1-12.

5 Banks RV, Campbell FW, Hood C. A neurophysiological approach to the treatment of amblyopia. $J$ Physiol 1978; 275: P 16-17.

6 Campbell FW, Hess RS, Watson PG, Banks R. Preliminary results of a physiologically based treatment of amblyopia. Br J Ophthalmol 1978; 62: 748-55.

7 Watson PG, Banks RV, Campbell FW, Hess RF. Clinical assessment of a new treatment for amblyopia. Trans Ophthalmol Soc UK 1978; 98: 201-8.

8 Pugh M. Foveal vision in amblyopia. Br J Ophthalmol 1954; 38: 321-31.

9 Pugh M. Visual distortion in amblyopia. Br J Ophthalmol 1958; 42: 449-60.

10 Flom MC, Weymouth FW, Kahneman D. Visual resolution and contour interaction. J Opt Soc Am 1963; 53: 1026-32.

11 Bailey IL, Lovie JE. New design principles for visual acuity letter charts. Am J Optom Physiol Opt 1976; 53: $740-5$.

12 Hess RF, Howell ER. The threshold contrast sensitivity function in strabismic amblyopia: evidence for a two type classification. Vision Res 1977; 17: 1049-57.

13 Hinchliffe HA. Clinical evaluation of stereopsis. $\mathrm{Br}$ Orthopt J 1978; 35: 46-57.

14 Dobson M. Binocular vision and the modern treatment of squint. London: Oxford University Press, 1933: 34-40.

15 Buchanan $\mathrm{D}$. The Revluc Amblyo-corrector. $\mathrm{Br} \mathrm{J}$ Physiolog Opt 1937; 11 : 43-4.

16 Arneson TJ. Some notes on fusion. Am J Optom Physiol Opt 1938; 15: 188-91.

17 Banks RV, Barnett F. A clinical assessment of new alternative methods of treatment of amblyopia. Proceedings 4th International Orthoptics Congress, Berne (in press). 